\title{
Quantum-Inspired Evolutionary Algorithm for Real and Reactive Power Dispatch
}

\author{
John G. Vlachogiannis and Kwang Y. Lee, Life Fellow, IEEE
}

\begin{abstract}
This paper presents an evolutionary algorithm based on quantum computation for bid-based optimal real and reactive power (P-Q) dispatch. The proposed quantum-inspired evolutionary algorithm (QEA) has applications in various combinatorial optimization problems in power systems and elsewhere. In this paper, the QEA determines the settings of control variables, such as generator outputs, generator voltages, transformer taps and shunt VAR compensation devices for optimal P-Q dispatch considering the bid-offered cost. The algorithm is tested on the IEEE 30-bus system, and the results obtained by the QEA are compared with those obtained by other modern heuristic techniques: ant colony system (ACS), enhanced GA and simulated annealing (SA) as well as the original QEA. Furthermore, in order to demonstrate the applicability of the proposed QEA, it is also implemented in a different problem, which is to minimize the real power losses in the IEEE 118-bus transmission system. The comparisons demonstrate an improved performance of the proposed QEA.
\end{abstract}

Index Terms-Bid-based dispatch, economic dispatch, evolutionary computation, quantum computation, real and reactive power operational planning.

\section{INTRODUCTION}

$\mathbf{N}$ EED for improved power quality and secure power supply today demands many services in the electric power systems, such as the dispatch of reactive power and other services known as ancillary services. Real and reactive power operational planning belongs to these categories of services. It allocates voltage controls and reactive power support in accordance with voltage security and open market mechanisms [1]. Many research works have revealed the coupling between real and reactive power support [1]-[8], while some others [9], [10] have tried to evaluate reactive power short-term marginal prices. Hao and Papalexopoulos proposed the development of local reactive power markets [11]. Zammit et al. designed ancillary service markets considering firstly security and secondly economic optimization in combination with spot market for electricity [12]. Recently, the discrete nature of the problem has led to the use of meta-heuristic techniques such as ant colony systems search

Manuscript received October 23, 2007; revised May 21, 2008. Current version published October 22, 2008. Paper no. TPWRS-00771-2007.

J. G. Vlachogiannis is with the Department of Electrical Engineering, Technical University of Denmark, Kgs. Lyngby, DK-2800, Denmark (e-mail: iv@elektro.dtu.dk).

K. Y. Lee is with the Department of Electrical and Computer Engineering, Baylor University, Waco, TX 76798-7356 USA (e-mail: Kwang_Y_ Lee@baylor.edu).

Color versions of one or more of the figures in this paper are available online at http://ieeexplore.ieee.org.

Digital Object Identifier 10.1109/TPWRS.2008.2004743
(ACS) [1], genetic algorithms (GAs) [13]-[19], and simulated annealing (SA) [20].

In this paper, the real and reactive operational planning is solved by means of a new quantum computing inspired genetic algorithm. Quantum computing was introduced in the early 1980s by Feynmann [21], [22] and Beinoff [23]. Quantum computers will operate on the superposition of all classical search states, allowing them to evaluate properties of all states in about the same time a classical machine requires for an evaluation of a single state. Superposition is described by a state vector, consisting of complex numbers, called amplitude amplifications [24]. Under these circumstances, quantum computing in the future could play a significant role in computer science. Recent researches (latest in the 1990s) look at quantum computing as a new evolutionary technique reducing the complexity of global optimization problems. They can be classified into two fields of studies: The first focuses on generating new quantum algorithms using evolutionary techniques such as genetic programming [25] and the second concentrates on quantum-inspired evolutionary computing for classical computers [26]-[31]. In the second field of studies, Han and Kim [29]-[31] introduced a quantum-inspired evolutionary algorithm (called the original QEA) when solving a class of combinatorial problems.

In this paper we propose an improved quantum-inspired evolutionary algorithm (QEA) for various combinatorial optimization problems applied in power engineering. The proposed QEA is backed by theoretical background, and characterized by rapid convergence and search capability compared with other classical GA and evolutionary techniques such as ACS algorithm [1]. These achievements are based on the concept of quantum theory such as qubits and a random superposition of quantum states. One individual in QEA can represent many states at the same time and there are weak relationships between individuals since each one of them is determined by current best solution and its probability, that is, the history of an individual up to date [24], [26]-[31].

Specifically, the QEA algorithm aims to determine the optimal settings of control variables, such as generator outputs, voltage magnitudes, transformer taps and the settings of shunt VAR compensation devices, which are considered in a quantum or Q-bit individual of QEA. Results are compared to those given by metaheuristic techniques of ACS search [1], enhanced GA [19], and SA [20] as well as the original QEA [29]-[31] for the network of IEEE 30-bus test system, and are shown to exhibit improved performance. Also, in order to demonstrate the applicability of the proposed QEA on a larger problem, we implement it to minimize the real power losses in transmission lines of the IEEE 118-bus system. The results are compared to those 
given by metaheuristics ACS search [1], enhanced GA [19], as well as the primal-dual interior-point based IP-OPF algorithm [41]. The full mathematical model of the IP-OPF [41] also is given in Appendix A. The last comparison demonstrates the excellent performance of proposed QEA on a larger optimization problem.

The rest of the paper is organized as follows: the problem of $\mathrm{P}-\mathrm{Q}$ dispatch is formulated in Section II. Section III addresses the fundamental concept of the quantum computation, while the QEA is introduced in Section IV. Section V presents the results of the QEA on bid-based real/reactive operational planning of the IEEE 30-bus system and minimization of real power losses in transmission lines of IEEE 118-bus system. Final conclusions with future works are outlined in Section VI.

\section{PROBlem Formulation}

The real and reactive power (P-Q) dispatch studied in this paper includes firstly, the economic resource allocation considering a typical bid structure and secondly, the security management. More specifically, the minimization of the offered bid cost, under the constraints of basic components and the steadystate transmission line loadings are examined [1]. The problem of real and reactive operational planning is formulated as an optimization problem with an objective function, expressed as [1], [20]

$$
C_{T}^{t}=\operatorname{Min} \sum_{i \in N g} C_{i}^{t}\left(P_{g i}^{t}\right)
$$

where $C_{T}^{t}$ is the total cost; $C_{i}^{t}$ is the active power bid cost of unit- $i$ at time- $t$; $P_{g i}^{t}$ is the real power generation of unit- $i$ at time- $t$; and $N g$ is the number of units, under the following equality and inequality constraints [1], [20].

\section{Equality constraints}

1) Typical load flow equations at buses.

Inequality constraints:

b) Generation constraints: Generator voltage magnitudes, real and reactive powers restricted by minimum and maximum limits.

c) Transformer constraints: Transformer taps bounded by minimum and maximum limits.

d) Shunt VAR constraints: Shunt VAR compensation restricted by its capacity.

e) Security constraints: Steady-state transmission line loadings.

The inequality constraints are incorporated in the objective function (1) as quadratic penalty terms [1], [20] as explained in Section V. The equality constraints are satisfied given that for each trial solution an ac power flow runs as explained in Section V.

\section{QUANTUM COMPUTING REPRESENTATION}

The basic concepts of quantum computing are addressed in this section [29]-[31]. The smallest unit of information stored in a two-state quantum computer is called a quantum bit or $Q$-bit. A Q-bit may be in the " 1 " state, in the "0" state, or in any superposition of the two, while a bit in traditional computing can

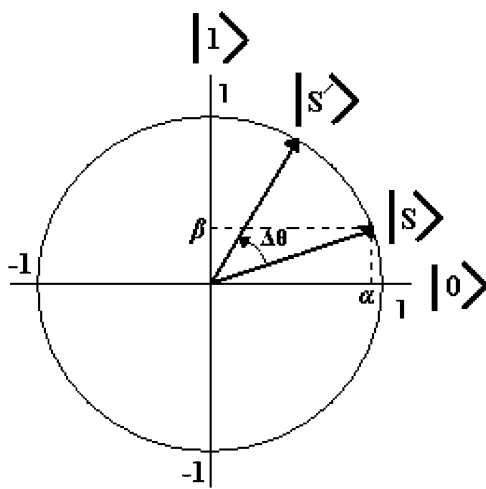

Fig. 1. Polar plot for qubits.

only hold a single state, either 0 or 1 [33]-[35]. The state of a Q-bit $\mathrm{S}$ can be represented as (Fig. 1)

$$
|\mathrm{S}\rangle=\alpha|0\rangle+\beta|1\rangle
$$

where $a$ and $\beta$ are complex numbers that specify the probability of the corresponding states; and therefore are called amplitude amplifications [31]. The $|\alpha|^{2}$ and $|\beta|^{2}$ give the probability that the Q-bit $|S\rangle$ will be found in the "0" and " 1 " states, respectively. So, they satisfy the relation: $|\alpha|^{2}+|\beta|^{2}=1$.

\section{A. Q-bit Individual Representation and Quantum Collapse}

If there is a system of Q-bits, the system can represent many states at the same time. A number of different representations can be used to encode the solutions onto individuals in an evolutionary computation. The representations can be classified broadly as: binary, numeric, and symbolic. The proposed QEA uses the representation, adopted in [29]-[31], called Q-bit for the probabilistic representation of the smallest unit of information and a Q-bit individual as a string of Q-bits, which are defined below.

A Q-bit individual with length $m$ is defined as a string of $m$ Q-bits

$$
q=\left[\begin{array}{c|c|c|c}
\alpha_{1} & \alpha_{2} & \ldots & \alpha_{m} \\
\beta_{1} & \beta_{2} & \ldots & \beta_{m}
\end{array}\right]
$$

where $\left|\alpha_{j}\right|^{2}+\left|\beta_{j}\right|^{2}=1,(j=1,2 \ldots, m)$.

The representation of the Q-bit individual has the advantage of representing a linear superposition of single states. In general the state of a Q-bit individual can be represented as

$$
\begin{aligned}
|S\rangle=A_{00 \ldots 0} \mid & 00 \ldots 0\rangle+A_{00 \ldots 1}|00 \ldots 1\rangle+\ldots \\
& +A_{01 \ldots 0}|01 \ldots 0\rangle+\ldots+A_{11 \ldots 1}|11 \ldots 1\rangle
\end{aligned}
$$

where $A_{00 \ldots 0}, A_{00 \ldots 1}, \ldots, A_{01 \ldots 1}, \ldots, A_{11 \ldots 1}$ are defined as the products

$$
\begin{aligned}
& A_{00 \ldots 0}=\alpha_{1} \alpha_{2} \ldots \alpha_{m}, \quad A_{00 \ldots 1}=\alpha_{1} \alpha_{2} \ldots \beta_{m}, \ldots \\
& A_{01 \ldots 0}=\alpha_{1} \beta_{2} \ldots \alpha_{m}, \quad \ldots A_{11 \ldots 1}=\beta_{1} \beta_{2} \ldots \beta_{m}
\end{aligned}
$$

Evolutionary computing with Q-bit individual representation has better characteristics of population diversity than other representations, since it can represent a superposition of the single states probabilistically. In classical computation, the 
possible states of a system of $m$ bits form a vector space of $m$ dimensions, i.e., we have $2^{m}$ possible single states. However, in a Q-bit individual of $m$ Q-bits the resulting state space has $2^{m}$ dimensions. It is this exponential growth of the state space with the number of particles that suggests a possible exponential speed-up of computation on quantum computers over classical computers. The measurement of a Q-bit individual projects the quantum state of Q-bit individual onto one of the single states associated with the measuring device. The result of a measurement is probabilistic and the process of measurement changes the state to that measured. This process is known as quantum collapse. Multi-measurements can be treated as a series of single Q-bit measurements (multi-quantum collapse) [33].

\section{B. Quantum Gates}

In the evolutionary computation with the Q-bit individual, the state of a Q-bit individual can be changed by the operation of a quantum gate. A quantum gate is a reversible gate and can be represented as a unitary operator $U$ acting on the quantum states $|\mathrm{S}\rangle$ satisfying $U^{+} U=U U^{+}$, where $U^{+}$is the Hermitian adjoint of $U$. There are several quantum gates, such as the NOT gate, controlled NOT gate, rotation gate, Hadamard gate, etc. [34]-[36]. For example, the NOT gate is the $2 \times 2$ matrix $\left(\begin{array}{ll}0 & 1 \\ 1 & 0\end{array}\right)$ and acts on a state of one Q-bits in a Q-bit individual (2)

$$
\begin{aligned}
U|\mathrm{~S}\rangle & =U \alpha|0\rangle+U \beta|1\rangle \\
& =\left(\begin{array}{ll}
0 & 1 \\
1 & 0
\end{array}\right) \alpha\left(\begin{array}{l}
1 \\
0
\end{array}\right)+\left(\begin{array}{ll}
0 & 1 \\
1 & 0
\end{array}\right) \beta\left(\begin{array}{l}
0 \\
1
\end{array}\right) \\
& =\alpha\left(\begin{array}{l}
0 \\
1
\end{array}\right)+\beta\left(\begin{array}{l}
1 \\
0
\end{array}\right)=\alpha|1\rangle+\beta|0\rangle=\operatorname{Not} \mathrm{S} .
\end{aligned}
$$

A rotation gate is employed to update each Q-bit in a Q-bit individual. The operation of the rotation gate $\mathbf{R}_{\mathbf{i}}$ on each Q-bit individual $\mathbf{q}_{\mathbf{i}}(\mathbf{t})$ in a population consisting of $n$ members/particles is presented as

$$
\left[\begin{array}{c}
\alpha_{j}(t+1) \\
\beta_{j}(t+1)
\end{array}\right]=\mathbf{R}_{i}(\mathbf{t}) \times\left[\begin{array}{c}
\alpha_{j}(t) \\
\beta_{j}(t)
\end{array}\right], \quad(j=1,2, \ldots, m)
$$

where $m$ is the number of Q-bits in the $i$ th Q-bit individual and the rotation gate is defined by

$$
\mathbf{R}_{i}(\mathbf{t})=\left[\begin{array}{cc}
\cos \Delta \theta_{i} & -\sin \Delta \theta_{i} \\
\sin \Delta \theta_{i} & \cos \Delta \theta_{i}
\end{array}\right], \quad(i=1,2, \ldots, n) .
$$

Here, $\Delta \theta_{i}$ is a rotation angle (see Fig. 1) of each Q-bit individual $\mathbf{q}_{\mathbf{i}}(\mathbf{t})$ toward either $\mid 0>$ or $\mid 1>$ state depending on its sign. The value of $\Delta \theta_{i}$ can be determined through a predetermined lookup table [30]. It can be also computed with the normalized difference (relative error) between objectives of each Q-bit individual $\mathbf{q}_{\mathbf{i}}(\mathbf{t})(i=1,2, \ldots, n)$ and the global best optimum (Q-bit individual b)

$$
\begin{aligned}
& \Delta \theta_{i}=\operatorname{sign}\left[\alpha_{b}(t)-\alpha_{j}(t)\right] \\
& \quad \cdot \operatorname{sign}\left[\alpha_{j}(t) \cdot \cos \Delta f_{i}+\beta_{j}(t) \cdot \sin \Delta f_{i}-\alpha_{j}(t)\right] \cdot \Delta f_{i} .
\end{aligned}
$$

Here, $\alpha_{j}, \beta_{j}$ represent the amplitude amplifications of $j$ th Q-bit in the $i$ th Q-bit individual; $b$ represents the global best
Q-bit individual in the population, and $\Delta f_{i}$ is the normalized difference (relative error) between objectives

$$
\Delta f_{i}=\pi \cdot\left(1-\frac{f(b)}{f\left(p_{i}(t)\right)}\right), \quad(i=1,2, \ldots, n)
$$

where $f\left(p_{i}(t)\right)$ and $f(b)$ are the objectives achieved by $i$ th Q-bit individual and the global best one (Q-bit individual $b$ ) in the population, respectively.

The sign function in (9) is defined by

$$
\operatorname{sign}[\eta]= \begin{cases}+1, & \text { if } \eta \geq 0 \\ -1, & \text { if } \eta<0 .\end{cases}
$$

The product of two sign functions in (9) indicates the direction of rotation $\Delta \theta_{i}$ so as for $\alpha_{j}(t+1)$ to be more close to $\alpha_{b}(t)$. Specifically, the first sign takes into account the difference between amplitude amplifications $\alpha_{j}(t)$ and $\alpha_{b}(t)$ and the second one the location of Q-bit individual (see Fig. 1). If it is located in the first/third quadrant the sign is set to positive $(+)$, else to negative (-).

These features give to the proposed QEA the possibility of a general EA applied in any combinatorial optimization problem. However, it can be specialized for the particular problem by choosing only the appropriate probability distribution of Q-bit individuals discussed in the next section.

\section{QUANTUM-InSPIRED EVOlutionARY ALGORITHM (QEA)}

In this section the QEA for bid-based real/reactive operational planning is presented. However, the QEA due to its general features, as it is presented, can be applied in any combinatorial optimization problem for power systems.

The main contribution of this QEA over the state-of-the art QEA [29]-[31] is the formula of the probability distribution that Q-bit individuals follow. In a quantum computer, the measurement of a quantum state results in its collapse to a single state, as described in the previous section [34]-[36]. However, collapsing into a single state does not occur in QEA, since QEA is working on a classical computer, rather than in a quantum computer. The process of quantum collapse in a classical computer is achieved by comparison of probabilities $\left|\alpha_{j}\right|^{2}$ or $\left|\beta_{j}\right|^{2}$ with random numbers $r_{j}$. These numbers can follow a probability distribution; for example, the uniform distribution, random $(0,1)$, as proposed in [29]-[31].

Let us assume that we have a little bit of information about the search space of the specific problem to be explored. Then, we can see that this prior knowledge can be easily put into use in generating the initial values of Q-bits in the population of Q-bit individuals [37]. After many empirical runs on real/reactive operational planning it is concluded that the knowledge of search space is better explored/exploited if random numbers $r_{j}$ follow the empirical formula of uniform distribution powered by $\ln (n \cdot m)$, where $n$ is the population size of Q-bit individuals and $m$ the number of Q-bits in the Q-bit individual. The product $n \cdot m$ represents the total length of all Q-bit individuals in the population. The empirical probability distribution formula increases the probability of Q-bits to be initialized/collapsed at the value of " 1 ". In this way, the area of search space with high 
values of control (decision) variables can be better explored/exploited. In other words, in this study, the global optimum solution is "hidden" near the "area" of search space, where there are high values of control variables than the rest of the space. Indeed, for instance large amounts of voltage magnitudes and shunt VAR compensation (respecting the operating constraints) increase the reactive power supply in the system resulting in the relief of generators and so the total cost of generator production is reduced. Consequently, the probability distribution formula that the quantum states of Q-bit individuals follow is

$$
r_{j}=\operatorname{random}(0,1)^{\ln (n \cdot m)}
$$

It is remarkable that the proposed probability distribution formula (12) followed by the collapsed Q-bits in this study has similar shape with the Plank radiation formula (probability distribution) followed by the density of neutrons and other elementary particles collapsed in the earth's gravitational field [38].

The steps of the QEA for real/reactive operational planning are described in the following paragraphs.

Step 1) For a given load pattern, set $t=0$.

Step 2) Translate each control variable (namely the permitted values of each one) to a Q-bit individual. If $m$ binary bits are needed to encode a Q-bit individual (which is determined depending on the desired accuracy), we will use a register of $m$ Q-bits to represent all the possible $2^{m}$ states of the quantum machine, namely the possible states in the feasible region of the optimization problem. In this study, there are four regions in each Q-bit individual, one for each set of control variables:

a) vector of generation PV bus voltage magnitudes, $\mathbf{V}_{\mathbf{G}}$;

b) vector of generator real output, $\mathbf{P}_{\mathbf{G}}$;

c) vector of transformer tap settings, $\mathbf{t}$; and

d) vector of bus shunt admittance, $\mathbf{b}_{\mathrm{SH}}$.

Encoding is performed using different Q-bit lengths for each set of control variables depending on the desired accuracy.

Step 3) Initialize a population of $n$ Q-bit individuals

$$
\mathbf{Q}(0)=\left\{\mathbf{q}_{1}(0), \mathbf{q}_{2}(0), \ldots \mathbf{q}_{n}(0)\right\}
$$

where each Q-bit individual, $\mathbf{q}_{i}(0), \quad(i=$ $1,2, \ldots, n)$, is with length $m$ ( $m$ Q-bits) as represented in (3).

Step 4) For all Q-bit individuals $\mathbf{q}_{i}(0),(i=1,2, \ldots, n)$ initialize the probabilities at

$$
\left[\begin{array}{l}
\alpha_{j}(0) \\
\beta_{j}(0)
\end{array}\right]=\left[\begin{array}{c}
r_{j} \\
\sqrt{1-r_{j}^{2}}
\end{array}\right], \quad(j=1,2, \ldots, m)
$$

where $r_{j}$ is the random variable generated by (12).

Step 5) While termination criterion (the maximum number of q-generations) is not reached, do
Begin

Step 5.1: $t=t+1$

Step 5.2 (Quantum collapse): Make $\mathbf{P}(t)$

solutions by observing quantum states

$|Q(t-1)\rangle$ as follows:

$\mathbf{P}(t)=\left\{p_{1}(t), p_{2}(t), \ldots p_{n}(t)\right\}$

Each $p_{j}(t)$ is the collapsed state of Q-bit individual $\mathbf{q}_{j}(t-1)$ and formed as an $m$-length classical GA chromosome or binary string. It includes $m$ binary bits ( 0 or 1 ), i.e., $p_{i}(t)$ is formed by selecting $m$ Q-bits

$$
p_{i}(t)=\left(p_{1} p_{2} \ldots p_{m}\right), \quad(i=1,2, \ldots, n) .
$$

Step 5.2.1: For each $p_{j}, j=1,2, \ldots, m$, generate a random number $r_{j}$ using (12). If $r_{j}<\beta_{j}(t)^{2},(j=1,2, \ldots, m)$ set $p_{j}$ at value of " 1 ", else " 0 ".

Step 5.2.2: Each $p_{i}(t)$ represents a candidate solution to the problem. So, evaluate each $p_{i}(t),(i=1,2, \ldots, n)$ running an ac power flow solution using the objective function $f\left(p_{i}(t)\right)$.

Step 5.3: Store the global best solution $f(b)$ (minimum in this case study) and the best Q-bit individual $b$ among $p_{i}(t),(i=1,2, \ldots, n)$. Step 5.4: Update each Q-bit individual $\mathbf{q}_{\mathbf{i}}(\mathbf{t})$ using the quantum operation $(U)$ of the rotation gate $\mathbf{R}_{\mathbf{i}}$ (7)-(11).

\section{RESUlts}

In the following case studies, the termination criterion of the proposed QEA which is the maximum number of q-generations is set at value of 500 .

\section{A. IEEE 30-Bus System}

The proposed QEA algorithm is tested on the real/reactive operational planning of IEEE 30-bus system and compared with those given by the ACS algorithm [1], enhanced GA [19], SA algorithm [20], and the original QEA [29]-[31].

The line diagram of the IEEE 30-bus network is given in Fig. 2. Some modifications in network's data were made as in [20] for comparison purposes. Specifically, the network consists of four generators, 41 lines, four transformers, and two capacitor banks (Fig. 2). For transformer taps, seven positions in each transformer were considered, each position corresponding to 0.02 increments within the interval $[0.94,1.06]$. The available reactive powers of capacitor banks are 0 MVAr, 7.5 MVAr, 15 MVAr, 22.5 MVAr, and 30 MVAr and they are connected to buses 10 and 24. Generator voltages were discretized in 150 steps (step: $0.0006 \mathrm{pu}$ ) within the range of $[0.96,1.05]$. For comparison purposes, load pattern is set at the values referred in [39], multiplied by a factor of 0.6 (nominal load). The accuracy for the generator outputs is set to $1 \mathrm{MW}$ (or $0.01 \mathrm{pu}$ ). 


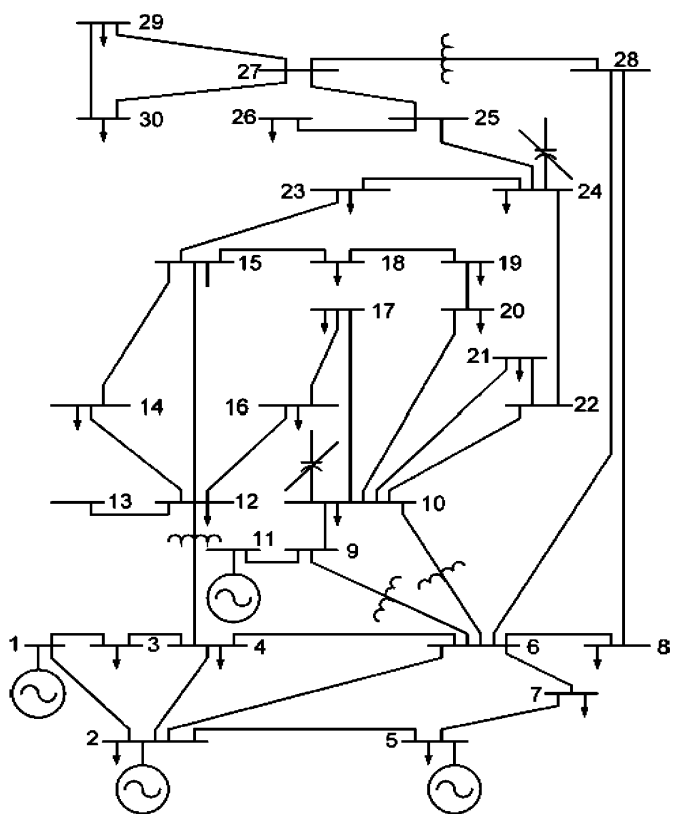

Fig. 2. Line diagram of the IEEE 30-bus system.

The bid curves of four generators are given by [20]

$\operatorname{bid}\left(\mathrm{P}_{1}\right)$

$$
=\left\{\begin{array}{l}
\text { 10euro/MWh, } 50 \mathrm{MW} \leq \mathrm{P}_{1}<80 \mathrm{MW} \\
\text { 20euro/MWh, } 80 \mathrm{MW} \leq \mathrm{P}_{1}<110 \mathrm{MW} \\
\text { 30euro/MWh, } 110 \mathrm{MW} \leq \mathrm{P}_{1}<140 \mathrm{MW} \\
\text { 40euro/MWh, } 140 \mathrm{MW} \leq \mathrm{P}_{1}<170 \mathrm{MW} \\
\text { 50euro/MWh, } 170 \mathrm{MW} \leq \mathrm{P}_{1} \leq 200 \mathrm{MW}
\end{array}\right\}
$$

$\operatorname{bid}\left(\mathrm{P}_{2}\right)$

$$
=\left\{\begin{array}{l}
\text { 10euro/MWh, } 20 \mathrm{MW} \leq \mathrm{P}_{2}<36 \mathrm{MW} \\
\text { 20euro/MWh, } 36 \mathrm{MW} \leq \mathrm{P}_{2}<52 \mathrm{MW} \\
\text { 30euro/MWh, } 52 \mathrm{MW} \leq \mathrm{P}_{2}<68 \mathrm{MW} \\
\text { 40euro/MWh, } 68 \mathrm{MW} \leq \mathrm{P}_{2}<84 \mathrm{MW} \\
\text { 50euro/MWh, } 84 \mathrm{MW} \leq \mathrm{P}_{2}<100 \mathrm{MW}
\end{array}\right\}
$$

$\operatorname{bid}\left(\mathrm{P}_{3}\right)$

$$
=\left\{\begin{array}{l}
\text { 10euro/MWh, } 10 \mathrm{MW} \leq \mathrm{P}_{3}<18 \mathrm{MW} \\
\text { 20euro/MWh, } 18 \mathrm{MW} \leq \mathrm{P}_{3}<26 \mathrm{MW} \\
\text { 30euro/MWh, } 26 \mathrm{MW} \leq \mathrm{P}_{3}<34 \mathrm{MW} \\
\text { 40euro/MWh, } 34 \mathrm{MW} \leq \mathrm{P}_{3}<42 \mathrm{MW} \\
\text { 50euro/MWh, } 42 \mathrm{MW} \leq \mathrm{P}_{3} \leq 50 \mathrm{MW}
\end{array}\right\}
$$

$\operatorname{bid}\left(\mathrm{P}_{4}\right)$

$$
=\left\{\begin{array}{l}
\text { 10euro/MWh, } 3 \mathrm{MW} \leq \mathrm{P}_{4}<5.4 \mathrm{MW} \\
\text { 20euro/MWh, } 5.4 \mathrm{MW} \leq \mathrm{P}_{4}<7.8 \mathrm{MW} \\
\text { 30euro/MWh, } 7.8 \mathrm{MW} \leq \mathrm{P}_{4}<10.2 \mathrm{MW} \\
\text { 40euro/MWh, } 10.2 \mathrm{MW} \leq \mathrm{P}_{4}<12.6 \mathrm{MW} \\
\text { 50euro/MWh, } 12.6 \mathrm{MW} \leq \mathrm{P}_{4} \leq 15 \mathrm{MW}
\end{array}\right\} .
$$

The binary length of each control variable is: 8 for each of the four generator voltages, namely $8 \times 4=32 \mathrm{Q}-$ bits for generator voltages; $8,7,6$, and 4 for each of the four generator outputs, namely $8+7+6+4=25 \mathrm{Q}-$ bits for generator outputs; 3 for each of the four transformer taps, namely $3 \times$ $4=12 \mathrm{Q}-$ bits for transformer taps; and 3 for each of the two capacitor banks, namely $3 \times 2=6 \mathrm{Q}-$ bits for capacitor banks. Therefore, the length of each Q-bit individual is $32+$

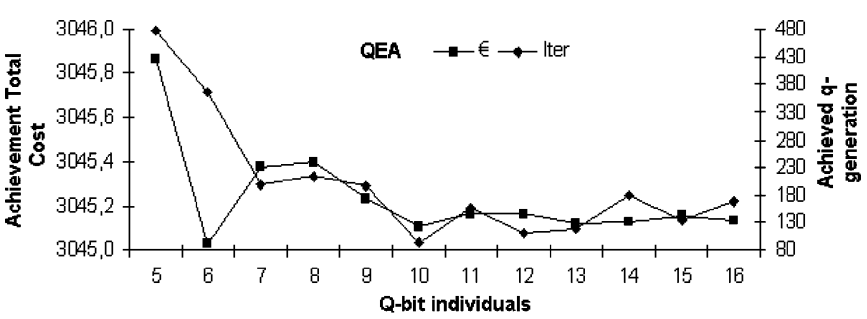

Fig. 3. Best achievement (euro) and achieved iterations of QEA algorithm (for 5 to 16 Q-bit individuals) in the nominal load of IEEE 30-bus system.

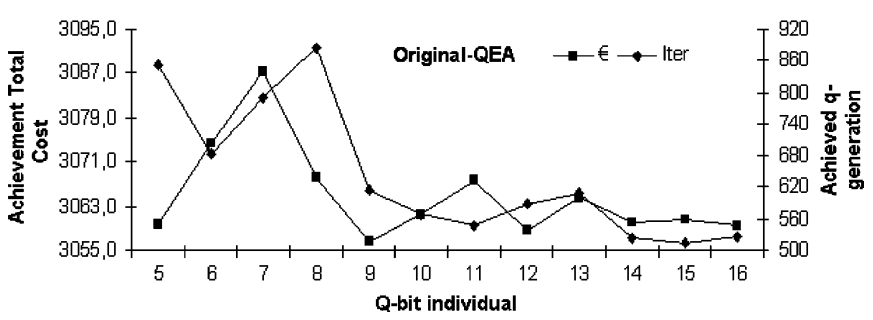

Fig. 4. Best achievement (euro) and achieved iterations of original-QEA algorithm (for 5 to 16 Q-bit individuals) in the nominal load of IEEE 30-bus system.

$25+12+6=75$. In other words, it consists of 75 Q-bits and can represent simultaneously $2^{75}$ binary states.

The objective function (1) is modified by augmenting it with constraints in the following formula [20]:

$$
\begin{aligned}
f=C_{T}^{t}+\sum_{i=1}^{N b}\left(V_{i}^{t}-V_{i}^{\text {max }}\right) & \times p(V)+\sum_{i=1}^{N b}\left(V_{i}^{\text {min }}-V_{i}^{t}\right) \times p(V) \\
& +\sum_{j=1}^{N l}\left(\frac{I_{j}^{t}}{I_{j}^{\text {max }}}-1\right) \times p(I)
\end{aligned}
$$

where $C_{T}^{t}$ expresses the total cost (1); $N b$ is the number of buses and $N l$ the number of lines; $V_{i}^{\min }, V_{i}^{\max }$ are the limits of voltage at bus- $i$, and $I_{j}^{\max }$ is the thermal limit of line- $j$.

The penalty factors $p(V)$ and $p(I)$ enforce the voltage and thermal limits, respectively [20]

$$
\begin{aligned}
& p(V)= \begin{cases}40, & \text { if } V_{i}^{t}>V_{i}^{\text {max }} \text { or } V_{i}^{t}<V_{i}^{\text {min }} \\
0, & \text { else }\end{cases} \\
& p(I)= \begin{cases}300, & \text { if } I_{j}^{t}>I_{j}^{\text {max }} \\
0, & \text { else. }\end{cases}
\end{aligned}
$$

Fig. 3 shows the convergence characteristics of proposed QEA. In particular, it shows the best achieved minimum solution of (21) and the corresponding number of q-generations achieved as a function of the number of Q-bit individuals in the population ranging from 5 to 16 Q-bit individuals. The number of Q-bit individuals between 5 and 16 gives the best compromise between satisfactory achievement and the total convergence time. From the figure it is concluded that the QEA with 6 Q-bit individuals (QEA\#6) achieves the best solution of 3045.03 euro in 366 iterations.

In order to see the contribution of the proposed QEA more clearly, the original QEA introduced in [29]-[31] was also tested and its convergence characteristics are shown in Fig. 4. From Fig. 4 it is concluded that the original QEA with 9 Q-bit individuals (original QEA\#9) achieves the solution of 3058.00 euro in 615 iterations. This result is better than the results obtained 


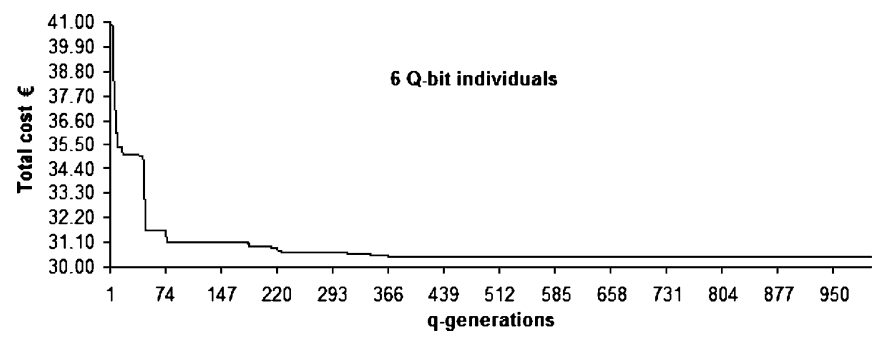

Fig. 5. Convergence of QEA\#6 in the nominal load of IEEE 30-bus system.

by the enhanced GA [19] and SA [20], but worse than the results of ACS [1] and the proposed QEA. The maximum number of q-generations of the original QEA was increased at value of 1000 .

The convergence characteristics of the proposed QEA\#6 are shown in Fig. 5. However, the QEA\#10 converges satisfactorily in the shortest time (Fig. 3). It converges in 94 iterations and the total CPU time is calculated at $0.261 \mathrm{~s}$. The ACS [1] converges in about 2000 iterations and the final value of the objective function (21) is estimated at 3050.2 euro [1]. The final values given by enhanced GA [19] and SA method are 3059.78 euro and 3110 euro [20], respectively. These are achieved in 686 and 150 iterations [20], respectively.

In conclusion, the proposed QEA performs better than ACS [1] and enhanced GA [19] in terms of convergence time. The SA [20] prematurely converges in 150 iterations. Also, the proposed QEA converges in fewer q-generations than those of the original QEA [29]-[31] achieving better results. Finally, the proposed QEA offers profit amount, which is calculated at 5.17 euro, 14.75 euro, 64.93 euro, and 12.97 euro, over other four EAs, respectively. The proposed QEA in contrast to other four EA requires only the regulation of the empirical formula (12). This formula could include a prior-knowledge that we have for the problem. The improved performance of the proposed QEA over the original one [29]-[31] is due to this formula which can better explore a search space.

Table I shows the values of control variables proposed by QEA\#6, original QEA\#9, ACS, enhanced GA and SA as well as their best achievements and number of achieved q-generations. These results confirm the prior-knowledge that the optimum solution was "hidden" in the "area" of search space with high values of control variables. Table I also gives the total CPU time for QEA, the original QEA, ACS and enhanced GA. The results of SA were obtained directly from [20] where there is no indication of the convergence time. The convergence time of QEA\#6 (0.855 s) is smaller than that of the original QEA\#9 [29]-[31] $(2.152 \mathrm{~s})$ and much smaller than that of ACS [1] (236.57 s) and the enhanced GA [19] (15.137 s). It is worthwhile to mention that in the case of ACS 300 artificial ants were competed. The enhanced GA's population size was taken equal to 80 , the maximum number of generations was 1000 , the length of chromosomes was 128 , and crossover and mutation were applied with initial probability 0.9 and 0.001 , respectively [19].

Finally, the bus voltages and apparent power flows in pu obtained by the proposed QEA are depicted in Figs. 6 and 7, respectively. The bus voltages are within the acceptable voltage
TABLE I

COMPARISON OF SETTINGS OF CONTROL VARIABLES FOR REAL/REACTIVE PLANNING OF IEEE 30-BUS SySTEM

\begin{tabular}{cccccc}
\hline $\begin{array}{c}\text { Control } \\
\text { Variables } \\
\begin{array}{c}\text { Objective } \\
\text { Convergence }\end{array}\end{array}$ & QEA & $\begin{array}{c}\text { Origin. } \\
\text { QEA }\end{array}$ & ACS & $\begin{array}{c}\text { Enhanced } \\
\text { GA }\end{array}$ & SA \\
\hline$P_{G I}(\mathrm{MW})$ & 79.503 & 79.501 & 108.51 & 108.989 & 107.998 \\
$P_{G 2}(\mathrm{MW})$ & 51 & 50 & 33 & 33 & 33 \\
$P_{G 5}(\mathrm{MW})$ & 33 & 32 & 25 & 25 & 25 \\
$P_{G I I}(\mathrm{MW})$ & 8 & 10 & 5 & 5 & 6 \\
$V_{G 1}$ & 1.050 & 1.060 & 1.037 & 1.033 & 1.000 \\
$\mathrm{~V}_{\mathrm{G} 2}$ & 1.050 & 1.060 & 1.022 & 1.018 & 1.020 \\
$\mathrm{~V}_{\mathrm{G} 5}$ & 1.050 & 1.060 & 1.012 & 1.008 & 1.010 \\
$\mathrm{~V}_{\mathrm{G} 11}$ & 1.050 & 1.060 & 1.041 & 1.034 & 1.020 \\
$T_{6-9}$ & 1.06 & 1.06 & 1.00 & 1.00 & 1.02 \\
$T_{6-10}$ & 1.06 & 0.96 & 0.94 & 0.94 & 0.98 \\
$\mathrm{~T}_{4-12}$ & 1.00 & 1.06 & 0.94 & 0.92 & 1.04 \\
$\mathrm{~T}_{27-28}$ & 0.96 & 0.96 & 1.02 & 1.00 & 0.98 \\
$Q C_{I}(\mathrm{MVAr})$ & 7.5 & 7.5 & 0.0 & 0.0 & 0.0 \\
$Q C_{2}(\mathrm{MVAr})$ & 7.5 & 0.0 & 15.0 & 15.0 & 7.5 \\
Total cost $(\boldsymbol{\epsilon})$ & $\mathbf{3 0 4 5 . 0 3}$ & $\mathbf{3 0 5 8 . 0}$ & $\mathbf{3 0 5 0 . 2}$ & $\mathbf{3 0 5 9 . 7 8}$ & $\mathbf{3 1 0 9 . 9 6}$ \\
Achieved $q-$ & 366 & 615 & 2010 & 686 & 150 \\
generation & & & & & \\
Total $C P U$ & 0.855 & 2.152 & 236.57 & 15.137 & - \\
(sec) & & & & & \\
\hline
\end{tabular}

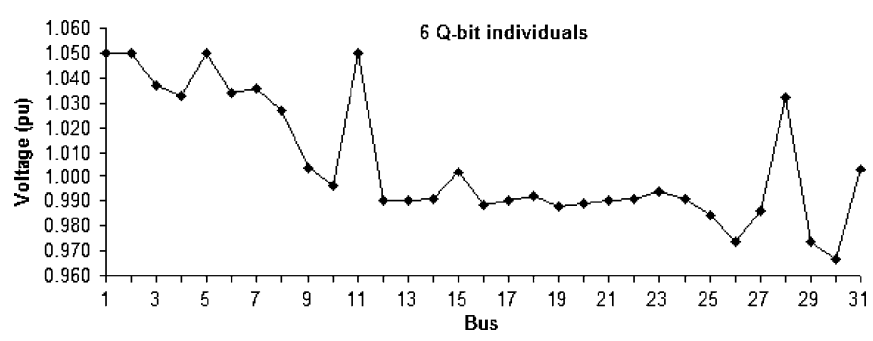

Fig. 6. Bus voltages (pu) of IEEE 30-bus system.

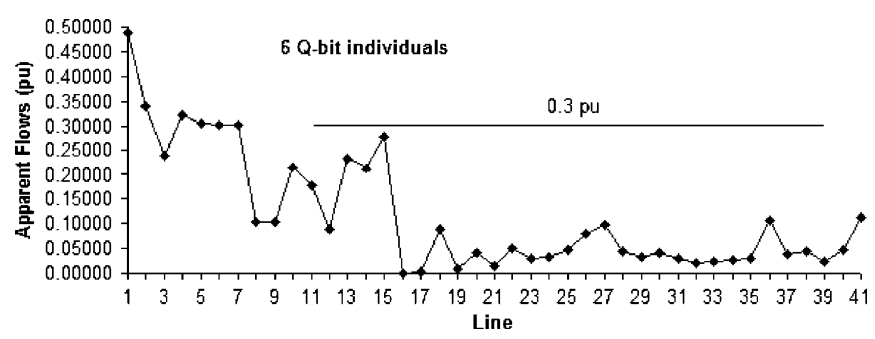

Fig. 7. Apparent power flows (pu) of IEEE 30-bus system.

range of $[0.96,1.05]$ as shown in Fig. 6. According to Fig. 7, all branch apparent power flows are much lower than the acceptable ranges of $202 \mathrm{MW}$ (for 132-kV lines between buses: 1-2, $1-3,2-4,2-5,2-6,3-4,4-6,6-28,5-7,6-8,6-7,8-28)$ and $30 \mathrm{MW}$ (for 33-kV lines) [20], [39].

\section{B. IEEE 118-Bus System}

In order to demonstrate the applicability of the proposed QEA on a larger power system, we implement it in minimizing the real power losses in transmission lines of the IEEE 118-bus system [40]. The results of QEA in this case study are compared with those given by ACS [1], the enhanced GA [19], and the classical primal-dual interior-point OPF algorithm (IP-OPF) (Appendix A). Specifically, above algorithms need to handle 


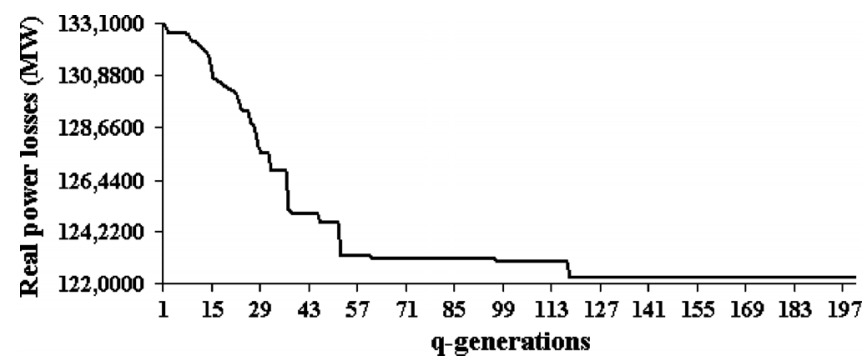

Fig. 8. Convergence of QEA\#80 in the nominal load of IEEE 118-bus system.

the problem of minimization of real power losses in transmission lines of IEEE 118-bus system satisfying physical and operational constraints. Such constraints are load flow equations, generator voltage limits and their reactive power outputs limits, switchable VAR compensations limits, transformer tap settings bounds and transmission line loadings. Specifically, the objective function is highly penalized (penalty factor: 1000) when the above constraints are violated. More details about this problem are given in [42].

In this case study the search space has 75 dimensions. Specifically QEA handles the real power generation of 54 generators, tap settings of nine transformers and reactive power injection of 12 capacitor banks. In the transformer tests, tap settings are considered within the interval $[0.9,1.1]$. The available reactive powers of capacitor banks are in $[0,30]$ MVAr. Voltages are considered within the range of $[0.95,1.1]$. The binary length of each control variable is set at: 14 for each one of 54 generator voltages, 12 for each one of nine transformer taps and 12 for each one of 12 capacitor banks. Since the binary length of voltage magnitudes at $\mathrm{PV}$ buses is $54 \times 14=756$, transformers tap settings is $9 \times 12=108$ and shunt VAR compensations is $12 \times 12=114$, the total length of each Q-bit individual is 1008 . In other words, one Q-bit individual consists of 1008 Q-bits and can represent simultaneously $2^{1008}$ binary states. Many runs of QEA have been done and it is found that the best population of Q-bit individuals in this study is 80 . The best population size of the enhance GA [19] is 100 chromosomes.

The proposed QEA\#80 converges in 118 q-generations achieving the least real power loss of 122.2227 MW (Fig. 8). The total CPU time is $12.592 \mathrm{~s}$ (Table II). The enhanced GA [19] achieves 131.9657 MW. It converges in 143 q-generations and the total CPU time is $15.375 \mathrm{~s}$ (Table II). The ACS [1] achieves $131.9010 \mathrm{MW}$. It converges in 1812 q-generations in the worst total CPU time of $532.90 \mathrm{~s}$ (Table II). The classical IP-OPF is the fastest algorithm since it converges within 4.068 $\mathrm{s}$ (eight iterations). However, it gives the worst solution of 132.1097 MW [42]. Due to the space limitation, Table II gives only 15 out of 75 values of decision variables as proposed in other methods. In this case study, the QEA performed remarkably well compared to other three methods. In all cases the total CPU time is calculated in a $1.4-\mathrm{GHz}$ Pentium-IV PC.

\section{CONCLUSIONS}

This paper presented an improved quantum-inspired evolutionary algorithm (QEA) for optimal P-Q dispatch in power systems. Although the proposed QEA has similar encoding
TABLE II

COMPARISON OF CONTROL VARIABLES FOR REAL POWER MINIMIZATION IN TRANSMISSION LINES OF IEEE 118-BUS SYSTEM

\begin{tabular}{|c|c|c|c|c|}
\hline $\begin{array}{c}\text { Control } \\
\text { Variables } \\
\text { Objective } \\
\text { Convergence } \\
\end{array}$ & QEA & ACS & $\begin{array}{c}\text { Enhanced } \\
\text { GA }\end{array}$ & IP-OPF \\
\hline$V_{G 1}$ & 1.05457 & 1.01687 & 1.02437 & 0.95500 \\
\hline$V_{G 4}$ & 1.06346 & 1.05270 & 1.02576 & 0.99800 \\
\hline$V_{G 34}$ & 1.05595 & 1.01955 & 1.02978 & 0.98400 \\
\hline$V_{G 65}$ & 1.05437 & 1.04786 & 0.99658 & 1.00500 \\
\hline$V_{G 92}$ & 1.06934 & 1.04645 & 1.01678 & 0.99000 \\
\hline$V_{G 113}$ & 1.07394 & 0.99906 & 1.03669 & 0.99300 \\
\hline$t_{8-5}$ & 0.99665 & 0.99133 & 0.99090 & 0.99726 \\
\hline$t_{26-25}$ & 1.10000 & 1.10000 & 1.10000 & 1.02806 \\
\hline$t_{30-17}$ & 0.99456 & 1.01476 & 1.01576 & 1.01440 \\
\hline$t_{68-69}$ & 0.90596 & 0.91905 & 0.94667 & 0.92988 \\
\hline$t_{81-80}$ & 0.91457 & 0.95797 & 0.92437 & 1.00317 \\
\hline$Q_{C 34}$ & 0.04358 & 0.20304 & 0.20564 & 0.18699 \\
\hline$Q_{C 48}$ & 0.12569 & 0.13747 & 0.17568 & 0.15299 \\
\hline$Q_{C 74}$ & 0.30000 & 0.26237 & 0.19700 & 0.20441 \\
\hline $\bar{Q}_{C 110}$ & 0.10000 & 0.13298 & 0.14234 & 0.16283 \\
\hline $\begin{array}{l}\text { Real power } \\
\text { losses }(M W)\end{array}$ & 122.2227 & 131.9010 & 131.9657 & 132.1097 \\
\hline $\begin{array}{l}\text { Achieved q- } \\
\text { generation }\end{array}$ & 118 & 1812 & 143 & 8 \\
\hline $\begin{array}{c}\text { Total CPU } \\
(\mathrm{sec})\end{array}$ & 12.592 & 532.90 & 15.375 & 4.068 \\
\hline
\end{tabular}

method with the classical GA, it can represent probabilistically much more states. Also its evolutionary mechanism is completely different and much more effective than the classical GA. The quantum population is progressed by the operation of simple quantum gates. Specifically, the QEA introduced a general quantum rotation gate applied to any combinatorial optimization problem. In addition, a prior knowledge for a specific problem was easily incorporated to the initial condition of QEA. This resulted in a new probability distribution that the collapsed Q-bits follow. The QEA was applied in P-Q dispatch of IEEE 30-bus system. The obtained results were compared with those obtained by Ant Colony Systems (ACS), the enhanced GA and Simulated Annealing (SA) as well as the original QEA algorithms, demonstrating an improved performance of QEA in finding the best solution within a satisfactory computing time. In order to demonstrate the performance of QEA we implement it on a larger problem, namely the minimization of real power losses in transmission lines of the IEEE 118-bus system. Results show an excellent performance of QEA over ACS, enhanced GA, and a conventional interior-point OPF-based algorithm.

\section{APPENDIX A}

The full mathematical model of the IP-OPF [41], which was already used in [42] is as following.

The objective function for the active power loss minimization problem is of the form

$$
\min P_{l o s s}(\mathbf{x})=\min \left(\sum P_{i j}+P_{j i}\right)
$$

where $P_{i j}$ is the active power flow from bus $i$ to bus $j$ [40], subject to

a) Equality constraints:

$$
\mathrm{g}(\mathbf{x})=0
$$


The vector $\mathbf{g}$ includes real and reactive power equations [40]. The vector $\mathbf{x}$ includes all state variables (voltage angles and magnitudes at buses) plus control variables (generator voltages, reactive powers of capacitor banks and active power generations). Also, we consider the loading parameter $\sigma$ as the last entry of the vector $\mathbf{x}$.

b) Inequality constraints:

$$
\mathbf{h}_{\min } \leq \mathbf{h}(\mathbf{x}) \leq \mathbf{h}_{\max }
$$

where vector $\mathbf{h}$ includes all [40]

i) Generation constraints: Generator voltage magnitudes, real and reactive powers restricted by minimum and maximum limits.

ii) Transformer constraints: Transformer taps bounded by minimum and maximum limits.

iii) Shunt VAR constraints: Shunt VAR compensation restricted by its capacity.

iv) Security constraints: Steady-state transmission line loadings.

The primal-dual IP method solves the perturbed Karush-Kuhn-Tucker (KKT) equations:

$$
\begin{aligned}
& \mathbf{g}(\mathbf{x})=0 \\
& \mathbf{s}+\mathbf{z}-\mathbf{h}_{\max }+\mathbf{h}_{\min }=0 \\
& \mathbf{h}(\mathbf{x})+\mathbf{z}-\mathbf{h}_{\max }=0 \\
& \nabla P_{\text {loss }}(\mathbf{x})+\mathbf{J}_{\mathbf{g}}(\mathbf{x})^{T} \cdot \boldsymbol{\lambda}+\mathbf{J}_{\mathbf{h}}(\mathbf{x})^{T} \cdot \mathbf{u}=0 \\
& \mathbf{S} \cdot \boldsymbol{\pi}=\mu \cdot \mathbf{e} \quad s \geq 0, \pi \geq 0 \\
& \mathbf{Z} \cdot \overline{\mathbf{u}}=\mu \cdot \mathbf{e} \quad z \geq 0, \widehat{u} \geq 0
\end{aligned}
$$

or for sort

$$
\mathbf{F}_{\mu}(\mathbf{y})=0, \quad \mathbf{y}=(\mathbf{s}, \mathbf{z}, \boldsymbol{\pi}, \overline{\mathbf{u}})
$$

where

$$
\overline{\mathbf{u}}=\mathbf{u}+\pi
$$

The $\mathbf{s}$ and $\mathbf{z}$ are slack vectors that transform inequalities into equalities; $\mathbf{S}$ and $\mathbf{Z}$ are diagonal matrices with $S_{i i}=s_{i}$ and $Z_{i i}=z_{i} ; \mu$ is a barrier parameter that is forced to go to 0 as $k$ (number of iterations) increases; $\mathbf{e}=(1,1, \ldots, 1)^{T}$ is a vector of ones of appropriate dimension (A8), (A9); $\mathbf{J}_{\mathrm{g}}$ is the Jacobian of $\mathbf{g} ; \mathbf{J}_{\mathbf{h}}$ is the Jacobian of $\mathbf{h}$; and $\nabla P_{\text {loss }}(\mathbf{x})$ is the gradient of real power losses (A1). The derivation of real power losses $P_{\text {loss }}(\mathbf{x})$ with respect to the loading parameter $\sigma$ (last entry of the vector $\mathbf{x}$ ) is given by

$$
\begin{aligned}
\frac{d P_{\text {loss }}(\mathbf{x})}{d \sigma}= & \sum_{l=1}^{\text {Nlines }}\left(\frac{d V_{i}}{d \sigma} \cdot V_{j}+\frac{d V_{j}}{d \sigma} \cdot V_{i}\right) \cdot g_{i j} \\
& \cdot\left(\cos \delta_{i j}+\cos \delta_{j i}\right)+2 \cdot V_{i} \cdot V_{j} \cdot g_{i j} \\
& \cdot \sin \delta_{j i} \cdot\left(\frac{d \delta_{i j}}{d \sigma}-\frac{d \delta_{j i}}{d \sigma}\right) \\
& -2 \cdot g_{i j}\left(\frac{d V_{i}}{d \sigma} \cdot V_{i}+\frac{d V_{j}}{d \sigma} \cdot V_{j}\right)
\end{aligned}
$$

The main steps of the IP-OPF algorithm are as follows:

Step 1) Set $k=0$, choose $\mu_{0}>0$ and a starting point $\mathbf{y}_{0}=\left(\mathbf{s}_{0}, \mathbf{z}_{0}, \boldsymbol{\pi}_{0}, \overline{\mathbf{u}}_{0}\right)>0$.

Step 2) Obtain the Newton system either (A10) or (A4)-(A9) at the current point $\mathbf{y}_{k}$ and solve for the Newton direction $\Delta \mathbf{y}$ using the fast re-factorization algorithm for block matrices $P R 2$ [43]:

$$
\mathbf{J}_{\mathbf{F}_{\mu}}\left(\mathbf{y}_{k}\right) \cdot \Delta \mathbf{y}=-\mathbf{F}_{\mu}\left(\mathbf{y}_{k}\right)
$$

where $\mathbf{J}_{\mathbf{F}_{\mu}}\left(\mathbf{y}_{k}\right)$ is the Jacobian of $\mathbf{F}_{\mu}$ evaluated at $\mathbf{y}_{k}$ and

$$
\mathbf{J}_{\mathbf{F}_{\mu}}\left(\mathbf{y}_{k}\right) \cdot \Delta \mathbf{y}=-\mathbf{F}_{\mu}\left(\mathbf{y}_{k}\right) .
$$

Step 3) Compute the maximum step length $a_{k} \in(0,1]$ in the direction of $\mathbf{y}_{k}$ :

$$
a_{k}=\min (1, \xi \cdot A)
$$

where $\xi \in(0,1)$ is a safety factor to ensure that $\mathbf{y}_{k+1}$ will hold the strict positive conditions $(\mathbf{s}, \mathbf{z}, \boldsymbol{\pi}, \overline{\mathbf{u}})>0$ and

$$
A=\min _{\Delta_{i}<0}\left(\frac{-s_{i}^{k}}{\Delta s_{i}}, \frac{-z_{i}^{k}}{\Delta z_{i}}, \frac{-\pi_{i}^{k}}{\Delta \pi_{i}}, \frac{-\bar{u}_{i}^{k}}{\Delta \bar{u}_{i}}\right)
$$

and obtain new estimates for the variables:

$$
\mathbf{y}_{k+1}=\mathbf{y}_{k}+\Delta \mathbf{y} .
$$

Step 4) If the point $\mathbf{y}_{k+1}$ satisfies the convergence criteria (A18)-(A20), then stop. If not, then set $k \leftarrow k+1$, reduce the barrier parameter $\mu_{k}$ using (A21), and return to Step 1.

Convergence criteria: The $k$ th iteration is considered converged if

$$
\max \left\{\begin{array}{l}
\max \left[\mathbf{h}_{\min }-\mathbf{h}\left(\mathbf{x}_{k}\right)\right], \\
\max \left[\mathbf{h}\left(\mathbf{x}_{k}\right)-\mathbf{h}_{\max }\right], \\
\left\|\mathbf{g}\left(\mathbf{x}_{\mathbf{k}}\right)\right\|
\end{array}\right\} \leq \varepsilon_{1}
$$

and

$$
\frac{\left\|\nabla P_{\text {loss }}\left(\mathbf{x}_{k}\right)+\mathbf{J}_{\mathbf{g}}\left(\mathbf{x}_{k}\right)^{T} \cdot \boldsymbol{\lambda}_{k}+\mathbf{J}_{\mathbf{h}}(\mathbf{x})^{T} \cdot \mathbf{u}_{k}\right\|_{\infty}}{1+\left\|\mathbf{x}_{k}\right\|_{2}+\left\|\boldsymbol{\lambda}_{k}\right\|_{2}+\left\|\mathbf{u}_{k}\right\|_{2}} \leq \varepsilon_{1}
$$

and

$$
\frac{\mathbf{S}_{k}^{T} \cdot \boldsymbol{\pi}_{k}+\mathbf{Z}_{k}^{T} \cdot \overline{\mathbf{u}}_{k}}{1+\left\|\mathbf{x}_{k}\right\|_{2}} \leq \varepsilon_{2}
$$

where $\varepsilon_{1}$ and $\varepsilon_{2}$ are predetermined tolerances. 
Reduction of the barrier parameter:

$$
\mu_{k+1}=\frac{\beta \cdot\left(\mathbf{S}_{k}^{T} \cdot \boldsymbol{\pi}_{k}+\mathbf{Z}_{k}^{T} \cdot \overline{\mathbf{u}}_{k}\right)}{2 \cdot p}
$$

where $\beta \in(0,1)$ is the expected decrease in residual of the complementary conditions: $\left(\mathbf{S}_{k}^{T} \cdot \boldsymbol{\pi}_{k}+\mathbf{Z}_{k}^{T} \cdot \overline{\mathbf{u}}_{k}\right)$ and $p$ is the number of inequality constraints.

\section{REFERENCES}

[1] K. Y. Lee and J. G. Vlachogiannis, "Ant colony optimization for active/reactive operational planning," in Proc. 16th IFAC World Congr., Prague, Czech Republic, Jul. 4-8, 2005, paper code: 6.3-02415.

[2] G. Gross, S. Tao, E. Bompard, and G. Chicco, "Unbundled reactive support service: Key characteristics and dominant cost component," IEEE Trans. Power Syst., vol. 17, no. 2, pp. 283-289, May 2002.

[3] E. L. Silva, J. Hedgecock, J. C. Mello, and J. C. Ferreira, "Practical cost based approach for the voltage ancillary service," IEEE Trans. Power Syst., vol. 16, no. 4, pp. 806-812, Nov. 2001.

[4] K. Bhattacharya and J. Zhong, "Reactive power as an ancillary service," IEEE Trans. Power Syst., vol. 16, no. 2, pp. 294-300, May 2001.

[5] N. H. Dandachi, M. J. Rawlins, O. Alsac, M. Prais, and B. Stott, "OPF for reactive pricing studies on the NGC system," IEEE Trans. Power Syst., vol. 11, no. 1, pp. 226-232, Feb. 1996.

[6] K. Y. Lee, J. L. Ortiz, M. A. Mohtadi, and Y. M. Park, "Optimal operation of large-scale power systems," IEEE Trans. Power Syst., vol. 3, no. 2, pp. 413-420, May 1988.

[7] K. Y. Lee, Y. M. Park, and J. L. Ortiz, "A united approach to optimal real and reactive power dispatch," IEEE Trans. Power App. Syst., vol. 104, no. 5, pp. 1147-1153, May 1985.

[8] K. Y. Lee, Y. M. Park, and J. L. Ortiz, "Fuel cost minimization for both real and reactive power dispatches," Proc. Inst. Elect. Eng. C, vol. 131, no. 1 , pp. 85-93, 1984.

[9] V. M. Dona and A. N. Peredes, "Reactive power pricing in competitive electric markets using the transmission losses function," in Proc. IEEE Power Tech. Conf., Porto, Portugal, Sep. 2001.

[10] A. A. El-Keib and X. Ma, "Calculating short-run marginal costs of active and reactive power production," IEEE Trans. Power Syst., vol. 12, no. 2, pp. 559-565, May 1997.

[11] S. Hao and A. Papalexopoulos, "Reactive power pricing and management," IEEE Trans. Power Syst., vol. 12, no. 1, pp. 95-104, Feb. 1997.

[12] M. A. B. Zammit, D. J. Hill, and R. J. Kaye, "Designing ancillary services markets for power system security," IEEE Trans. Power Syst., vol. 15, no. 2, pp. 675-680, May 2000.

[13] Y.-L. Chen, "Weak bus-oriented optimal multi-objective VAR planning," IEEE Trans. Power Syst., vol. 11, no. 4, pp. 1885-1890, Nov. 1996.

[14] K. P. Wong and Y. W. Suzannah, "Combined genetic algorithm/simulated annealing/fuzzy set approach," IEEE Trans. Power Syst., vol. 11, no. 1, pp. 128-136, Feb. 1996.

[15] Y.-T. Yang, P.-C. Yang, and C.-L. Huang, "Evolutionary programming based economic dispatch for units with non-smooth fuel cost functions," IEEE Trans. Power Syst., vol. 11, no. 1, pp. 112-118, Feb. 1996.

[16] K. Y. Lee, X. Bai, and Y. M. Park, "Optimization method for reactive power planning using a genetic algorithm," IEEE Trans. Power Syst., vol. 10, no. 4, pp. 1843-1850, Nov. 1995.

[17] Y.-L. Chen and C.-C. Liu, "Optimal multi-objective VAR planning using an interactive satisfying method," IEEE Trans. Power Syst., vol. 10, no. 2, pp. 664-670, May 1995.

[18] Y.-T. Hsiao, H.-D. Chiang, C.-C. Liu, and Y.-L. Chen, "A computer package for optimal multi-objective VAR planning in large scale power systems," IEEE Trans. Power Syst., vol. 9, no. 2, pp. 668-676, May 1994.

[19] A. G. Bakirtzis, P. N. Biskas, C. E. Zoumas, and V. Petridis, "Optimal power flow by enhanced genetic algorithm," IEEE Trans. Power Syst., vol. 17, no. 2, pp. 229-236, May 2002.

[20] K. A. Papadogiannis, N. D. Hatziargyriou, and J. T. Saraiva, "Bid based coupled active/reactive dispatch using simulated annealing," Eng. Intell. Syst., vol. 12, no. 3, pp. 175-184, 2004.

[21] R. P. Feynmann, "Simulating physics with computers," Int. J. Theoret. Phys., vol. 21, pp. 467-482, 1982.

[22] R. P. Feynmann, "Quantum mechanical computers," Found. Phys., vol. 16 , no. 6 , pp. $507-531,1986$.
[23] P. Beinoff, "The computers as a physical system: A microscopic of Hamiltonian model of computers as presented by Turing machines," $J$. Statist. Phys., vol. 22, pp. 563-591, 1980.

[24] T. Hogg and D. Portnov, "Quantum optimization," Inf. Sci., vol. 128, pp. 181-197, 2000

[25] L. Spector, H. Barnum, H. J. Bernstein, and N. Swamy, "Finding a better-than-classical quantum AND/OR algorithm using genetic programming," in Proc. 1999 Congr. Evolutionary Computation, Jul. 1999, pp. 2239-2246.

[26] B. Rylander, T. Soule, J. A. Foster, and J. Alves-Foss, "Quantum evolutionary programming," in Proc. Int. Conf. Genetic and Evolutionary Computation (GECCO), 2001, pp. 1005-1011, Morgan Kaufmann.

[27] A. Malossini, E. Blanzieri, and T. Calarco, QGA: A Quantum Genetic Algorithm, Trento, Italy, Tech. Rep. DIT-04-105, 2004. [Online]. Available: http://www.dit.unitn.it.

[28] A. Narayanan and M. Moore, "Quantum-inspired genetic algorithms," in Proc. IEEE Int. Conf. Evolutionary Computation, 1996, pp. 61-66.

[29] K.-H. Han and J.-H. Kim, "Quantum-inspired evolutionary algorithms with a new termination criterion, He Gate, and two-phase scheme," IEEE Trans. Evol. Comp., vol. 8, no. 2, pp. 156-169, Apr. 2004.

[30] K.-H. Han and J.-H. Kim, "Quantum-inspired evolutionary algorithm for a class of combinatorial optimization," IEEE Trans. Evol. Comp., vol. 6 , no. 6, pp. 580-593, Dec. 2002.

[31] K.-H. Han and J.-H. Kim, "Genetic quantum algorithm and its application to combinatorial optimization problem," in Proc. 2000 Congr. Evolutionary Computation, Jul. 2000, pp. 1354-1360.

[32] G.-X. Zhang, N. Li, W.-D. Jin, and L.-Z. Hu, "Novel quantum genetic algorithm and its applications," Front. Elect. Electron. Eng., vol. 1, pp. 31-36, 2006, translated from Acta Electronica Sinica, 2004, vol. 32, no. 3, pp. 476-479 (in Chinese).

[33] A. Draa, M. Batouche, and H. Talbi, "A quantum-inspired differential evolution algorithm for rigid image registration," Enformatika Trans. Eng., Comp., Tech., vol. VI, pp. 408-411, Dec. 2004.

[34] G. Cybenco, "Reducing quantum computations to elementary unitary operations," IEEE Comput., pp. 27-32, Apr./May 2001.

[35] T. Hey, "Quantum computing: An introduction," Comput. Control Eng. $J$. , vol. 10, no. 3, pp. 105-112, 1999, Piscataway, NJ: IEEE Press.

[36] A. Barenco, C. H. Bennett, R. Cleve, D. P. Di Vincenzo, N. Margolus, P. Shor, T. Sleator, J. A. Smolin, and H. Weinfurter, "Elementary gates for quantum computation," Phys. Rev. A, vol. 52, pp. 3457-3467, 1995.

[37] D. Ventura and T. Martinez, "Initializing the amplitude distribution of a quantum state," Found. Phys. Lett., vol. 12, no. 6, pp. 547-559, 1999.

[38] V. V. Nesvizhevsky, A. K. Petukhov, H. G. Borner, T. A. Baranova, A M. Gagarski, G. A. Petrov, K. V. Protasov, A. Y. Voronin, S. Baessler, H. Abele, A. Westphal, and L. Lucovac, "Investigation of the neutron quantum states in the earth's gravitational field," J. Res. Nat. Inst. Stand. Technol., vol. 110, no. 3, pp. 263-267, 2005.

[39] [Online]. Available: http://www.ee.washington.edu/research/pstca/ pf30/pg_tca30bus.htm.

[40] [Online]. Available: http://www.ee.washington.edu/research/pstca/ pf118/pg_tca118bus.htm.

[41] A. C. Z. de Souza, L. M. Honorio, G. L. Torres, and G. Lambert-Torres, "Increasing the loadability of power systems through optimal-localcontrol actions," IEEE Trans. Power Syst., vol. 19, no. 1, pp. 188-194, Feb. 2004.

[42] J. G. Vlachogiannis and K. Y. Lee, "A comparative study on particle swarm optimization for optimal steady-state performance of power systems," IEEE Trans. Power Syst., vol. 21, no. 4, pp. 1718-1728, Nov. 2006.

[43] A. G. Bakirtzis and J. G. Vlachogiannis, "Block matrices and their applications in power systems," Elect. Power Syst. Res., vol. 28, no. 1, pp. $51-58,1993$.

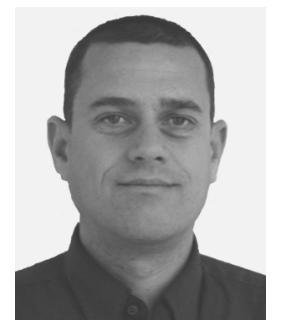

John G. Vlachogiannis received the B.Sc. degree in electrical engineering and Ph.D. degree from the Aristotle University of Thessaloniki, Thessaloniki, Greece, in 1990 and 1994, respectively.

$\mathrm{He}$ is an Associate Professor at the Department of Electrical Engineering of the Technical University of Denmark (DTU), Kgs. Lyngby. Also he has joined with the Research Centre for Electric Technology (CET) of DTU. His research interests include control and management strategies and artificial intelligence techniques in planning and operation of power and

industrial systems.

Dr. Vlachogiannis is a member of the Greek Computer Society (Member of IFIP, CEPIS) and a member of the Technical Chamber of Greece. 


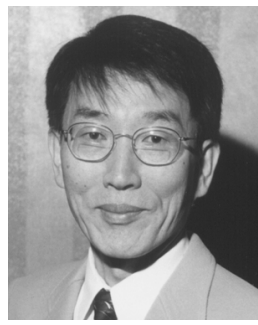

Kwang Y. Lee (LF'08) received the B.S. degree in electrical engineering from Seoul National University, Seoul, Korea, in 1964, the M.S. degree in electrical engineering from North Dakota State University, Fargo, in 1968, and the Ph.D. degree in system science from Michigan State University, East Lansing, in 1971.

He has been with Michigan State, Oregon State, the University of Houston, the Pennsylvania State University, and Baylor University, where he is now a Professor and Chairman of the Electrical and Computer Engineering and Director of the Power and Energy Systems Laboratory. His interests include power system control, operation, planning, and computational intelligence for power systems and power plant control.

Dr. Lee is a former Associate Editor of IEEE TRANSACTIONS ON NEURAL NETWORKS and Editor of IEEE TRANSACTIONS ON ENERGY CONVERSION. He is also a registered Professional Engineer. 